\title{
Evaluation De L'efficacité De Fongicides Au Laboratoire Contre Corynespora Cassiicola, Agent Causal De La Maladie « Corynespora Leaf Fall » De L'hévéa En Côte d'Ivoire
}

\author{
Yao Kouakou Alban Prosper, \\ Université Nangui Abrogoua, Côte d'Ivoire \\ Centre National de Recherche Agronomique, Côte d'Ivoire. \\ Wahounou Polié Jean, \\ Centre National de Recherche Agronomique, Côte d'Ivoire.
}

Diallo Atta Hortense,

Université Nangui Abrogoua, Côte d'Ivoire.

Doi: 10.19044/esj.2018.v14n18p340 URL:http://dx.doi.org/10.19044/esj.2018.v14n18p340

\begin{abstract}
Corynespora leaf fall disease (CLFD) caused by Corynespora cassiicola is increasingly a major problem for the development of rubber production in Côte d'Ivoire. In the search for solutions to this problem, the efficacy of 11 fungicides (Azoxystrobin, Carbendazime + Chlorothalonil, Carbendazime, Chlorothalonil, Difenoconazole, Fosetyl-Aluminum, Iprodione, Mancozeb, Metalaxyl + Copper Oxide, Pyraclostrobin + Fenpropimorph, Triadimefon) was laboratory tested against an isolate of $C$. cassiicola. The results of this test showed a high level of efficacy of four fungicides, Iprodione, Carbendazime + Chlorothalonil, Carbendazim and Pyraclostrobin + Fenpropimorph. The use of these four fungicides formulations in mature plantation would be well indicated, to overcome this foliar pathology.
\end{abstract}

Keywords: Corynespora cassiicola, CLFD, hevea, fungicides, Côte d'Ivoire

Résumé

La maladie de chute des feuilles «Corynespora leaf fall ou CLF » causée par Corynespora cassiicola constitue de plus en plus un problème majeur pour le développement de l'hévéaculture en Côte d'Ivoire. Dans la recherche de solutions à ce problème, l'efficacité de 11 fongicides (Azoxystrobine, Carbendazime + Chlorothalonil, Carbendazime, Chlorothalonil, Difenoconazole, Fosetyl - Aluminium, Iprodione, 
Mancozèbe, Metalaxyl + Oxyde de cuivre, Pyraclostrobine + Fenpropimorphe, Triadimefon) a été testée au laboratoire contre un isolat de C. cassiicola. Les résultats des tests in vitro ont montré un haut niveau d'efficacité de quatre fongicides, Iprodione, Carbendazime + Chlorothalonil, Carbendazime et Pyraclostrobine + Fenpropimorphe. L'emploi de ces quatre formulations fongicides en plantation mature serait bien indiqué, pour venir à bout de cette pathologie foliaire.

Mots clés : Corynespora cassiicola, CLF, hévéa, fongicides, Côte d'Ivoire

\section{Introduction}

Corynespora cassiicola (Berk \& Curt.) Wei. est un agent pathogène fongique responsable de la maladie de chute des feuilles (CLF) chez l'hévéa. Cette maladie, comme son nom l'indique, entraine des défoliations répétées qui affaiblissent l'arbre et par conséquent sa production à cause de la perturbation de la photosynthèse. Elle a été découverte pour la première fois en Inde en 1958 (Ramakrishan et Pillay, 1961). Initialement confinée en pépinières, elle s'est ensuite étendue aux jeunes puis aux arbres adultes dans certaines localités de l'Inde au cours des années 1969 à 1976 (Georges et Edathil, 1980). Elle fit son apparition en Malaisie en 1960 (Newsam, 1961), au Nigeria en 1969 (Awederu, 1969), en Indonésie en 1980 (Teoh, 1983), au Brésil (Junqueira et al., 1985), au Sri lanka (Liyanage et al., 1986) et en Thaïlande en 1985 (Pongthep, 1987). Considéré au départ comme étant un champignon facultatif, $C$. cassiicola est devenu aujourd'hui une véritable menace pour l'industrie du caoutchouc mondial (Jayasinghe, 2000). Cette affection pathologique se caractérise par des lésions nécrotiques développées sur les feuilles avec un noircissement des nervures donnant une apparence typique d'《 arête de poisson » ou « rail de train » (Ramakrishanan et Pillay, 1961). Elle se manifeste toute l'année et affecte les feuilles à tous les stades de développement. L'infection d'un clone sensible entraîne une défoliation complète et parfois la mort de l'arbre (Kajornckaikul, 1987). Dans de nombreux cas, elle provoque de sérieuses pertes de la production de caoutchouc naturel d'environ 20 à $25 \%$ (Barthe et $a l ., 2007$ ).

En Côte d'Ivoire, de fortes attaques de Corynespora cassiicola ont été signalées pour la première fois, sur les clones RRIC 103 et RRIC 110, dans les années 90 ; entraînant ainsi leur retrait de la liste des clones recommandés (Wahounou et al., 1996). Cette mesure visait à éviter la propagation de la maladie sur l'ensemble du verger hévéicole en l'absence de moyens de lutte. Depuis 2010, il y a la résurgence des attaques épidémiques sur le clone IRCA 18 avec d'importantes conséquences économiques. Compte tenu de l'ampleur des dégâts causés par $C$. cassiicola sur les hévéas, notamment sur le clone sensible IRCA 18 qui couvre environ $10 \%$ du verger hévéicole national, la 
recherche de moyen de lutte contre ce champignon devient une nécessité. Ce, d'autant plus que la liste des clones sensibles pourrait s'allonger, aggravant ainsi la détresse des hévéacultures. Avec les avancées en matière d'élaboration de molécules chimiques davantage efficaces et à effet sur le court terme, la lutte chimique s'impose dans une première étape dans les investigations pour faire face à ce fléau.

Ce présent travail rend compte d'une évaluation au laboratoire de l'efficacité de fongicides contre $C$. cassiicola afin de rechercher un moyen de lutte efficace contre ce champignon.

\section{Matériels et méthodes}

\section{Matériel fongique}

L'agent fongique utilisé dans cette étude a été un isolat de Corynespora cassiicola isolé sur du milieu de culture PDA, à partir de feuilles d'hévéa malade échantillonnées dans une plantation d'Aboisso, région située dans le Sud-est de la Côte d'Ivoire.

\section{Fongicides}

Les fongicides utilisés dans cet essai sont au nombre de onze. Il s'agit d'Azoxystrobine, Carbendazime + Chlorothalonil, Carbendazime, Difenoconazole, Fosetyl - Aluminium, Iprodione, Mancozèbe, Metalaxyl + Oxyde de cuivre, Pyraclostrobine + Fenpropimorphe, Triadimefon. Ces fongicides ont été offerts par les firmes phytosanitaires de Côte d'Ivoire. Leurs caractéristiques physico-chimiques sont résumées dans le tableau ci-dessous.

Tableau 1 : Caractéristiques des fongicides utilisés contre Corynespora cassiicola

\begin{tabular}{lllll}
\hline $\begin{array}{l}\text { Nom } \\
\text { commercial }\end{array}$ & Matière active & Concentration & $\begin{array}{l}\text { Type de } \\
\text { formulation }\end{array}$ & Mode d'action \\
\hline Balear & Chlorothalonil & $720 \mathrm{~g} / \mathrm{l}$ & $\begin{array}{l}\text { Suspension } \\
\text { concentrée }\end{array}$ & Contact \\
Bankit & Azoxystrobine & $250 \mathrm{~g} / \mathrm{l}$ & $\begin{array}{l}\text { Suspension } \\
\text { concentrée }\end{array}$ & Systémique \\
Banko plus & Carbendazime & $100 \mathrm{~g} / \mathrm{l}$ & $\begin{array}{l}\text { Suspension } \\
\text { concentrée }\end{array}$ & Systémique \\
Calliète & Chlorothalonil & $550 \mathrm{~g} / 1$ & $\begin{array}{l}\text { Concentré } \\
\text { Cómulsionnable }\end{array}$ & Systémique \\
Carhino & Fosetyl- Aluminium & $800 \mathrm{~g} / \mathrm{kg}$ & $\begin{array}{l}\text { Poudre mouillable } \\
\text { Concentré }\end{array}$ & Systémique \\
Comet Plus & Carbendazime & $500 \mathrm{~g} / \mathrm{kg}$ & $\begin{array}{l}\text { émulsionnable } \\
\text { Concentré }\end{array}$ & Systémique \\
Difecor & Pyraclostrobine & $100 \mathrm{~g} / \mathrm{l}$ & émulsionnable \\
Ivory & Fenpropimorphe & $375 \mathrm{~g} / 1$ & Poudre mouillable & Constémique \\
Piécho & Difenoconazole & $250 \mathrm{~g} / 1$ & $\begin{array}{l}\text { Poudre mouillable } \\
\text { Concentré }\end{array}$ & Contact \\
Trical & Mancozèbe & $800 \mathrm{~g} / \mathrm{kg}$ & émulsionnable & Systémique \\
Ridomil plus & Iprodione & $500 \mathrm{~g} / \mathrm{kg}$ & Poudre mouillable & Systémique \\
\hline
\end{tabular}




\section{Evaluation de la sensibilité in vitro de Corynespora cassiicola aux fongicides}

Dans cette étude, la technique d'appât empoisonné a été utilisée pour évaluer les différents fongicides. Le milieu de culture PDA composé de flocons de pomme de terre (20 g/l), D-glucose $(20 \mathrm{~g} / \mathrm{l})$ et d'Agar-Agar $(20 \mathrm{~g} / \mathrm{l})$ a été utilisé comme milieu de base. Après autoclavage des milieux, des quantités de chaque fongicide ont été prélevées puis incorporées au milieu PDA, donnant ainsi, cinq niveaux de concentration : 10, 50, 100, 500 et 1000 $\mathrm{ppm}$. Une fois le milieu amendé, des disques mycéliens de $1 \mathrm{~cm}$ de diamètre prélevés au front de croissance des cultures pures de C. cassiicola âgées de 7 jours, ont été déposés au centre des milieux solidifiés incorporés des différentes concentrations de fongicides (traitements) et des milieux solidifiés sans fongicide (témoin). Pour chaque traitement, cinq boîtes de Pétri ont été utilisées. L'expérience a été répétée 3 fois.

Des mesures journalières du diamètre mycélien des colonies fongiques ont été effectuées à l'aide d'une règle graduée suivant deux axes perpendiculaires au revers de la boîte de Pétri. Les mesures ont été arrêtées lorsque les filaments mycéliens ont atteint la périphérie de la boîte de Pétri de $9 \mathrm{~cm}$ de diamètre dans les lots témoins. L'efficacité des fongicides a été évaluée selon la méthode Kumar et al. (2007). Pour chaque concentration, le taux d'inhibition moyen de la croissance mycélienne a été calculé selon la formule proposée par Vincent (1927).

$$
\mathrm{I}(\%)=100(\mathrm{D} 0-\mathrm{DT}) / \mathrm{DO}
$$

I (\%) : Taux d'inhibition en pourcentage du diamètre mycélien

$\mathrm{D}_{0}$ : Diamètre mycélien $(\mathrm{cm})$ des colonies témoins

$\mathrm{D}_{\mathrm{T}}$ : Diamètre mycélien $(\mathrm{cm})$ des colonies traitées

Le niveau de sensibilité ou de résistance de l'isolat aux fongicides a été déterminé selon l'échelle de Kumar et al. (2007) avec de légères modifications (tableau 2).

Tableau 2 : Niveau d'efficacité fongicide en fonction de l'échelle de Kumar et al. (2007)

\begin{tabular}{llll}
\hline Echelle & $\begin{array}{l}\text { Classe des taux } \\
\text { d'inhibition } \\
\text { mycélienne I }(\%)\end{array}$ & $\begin{array}{l}\text { Niveau de } \\
\text { sensibilité/résistance du } \\
\text { champignon }\end{array}$ & $\begin{array}{l}\text { Niveau d'efficacité du } \\
\text { fongicide }\end{array}$ \\
\hline 1 & $\mathrm{I}>90 \%$ & Hautement Sensible & Très bonne efficacité \\
2 & $75 \%<\mathrm{I} \leq 90 \%$ & Sensible & Bonne efficacité \\
3 & $60 \%<\mathrm{I} \leq 75 \%$ & Modérément Résistant & Efficacité moyenne \\
4 & $40 \% \leq \mathrm{I} \leq 60 \%$ & Résistant & Faible efficacité \\
5 & $\mathrm{I}<40 \%$ & Hautement Résistant & Très faible efficacité \\
\hline
\end{tabular}




\section{Analyses statistiques}

Les taux d'inhibition de la croissance mycélienne ont subi une transformation $\arcsin \sqrt{ }$ en vue de normaliser la distribution et égaliser les variances. Une analyse de variance (ANOVA) à $5 \%$ de signification a été effectuée à l'aide du logiciel informatique Statistica version 7.1. La comparaison des moyennes des taux d'inhibition a été effectuée avec le test de Newman et Keuls au seuil de $5 \%$.

\section{Résultats}

\section{Efficacité in vitro des fongicides contre Corynespora cassiicola}

Le test d'évaluation au laboratoire de l'efficacité de 11 fongicides contre Corynespora cassiicola par la technique d'appât empoisonné montre globalement que, l'effet des fongicides sur la croissance mycélienne du champignon varie en fonction du fongicide et des doses utilisées (Figure 1).

En effet, sur les milieux de culture contenant Iprodione et Pyraclostrobine + Fenpropimorphe, aucun développement mycélien de $C$. cassiicola n'a été observé, même à faible dose. A partir de 50 ppm, aucune croissance fongique n'a été observée au niveau de Carbendazime + Chlorothalonil, Carbendazime et Mancozèbe. Avec 10 ppm, la croissance mycélienne a été très faible pour Carbendazime + Chlorothalonil et Carbendazime, avec 0,3 et $0,4 \mathrm{~cm}$ de diamètre et moyenne pour Mancozèbe $(4,65 \mathrm{~cm})$. Quelle que soit la dose (10, 50, 100, 500 et 1000 ppm), les fongicides utilisés, à l'exception de Métalaxyl + Oxyde de cuivre, se distinguent statistiquement les uns des autres par la croissance mycélienne et sont différents du témoin (croissance mycélienne sur milieu sans fongicide).

L'échelle de Kumar et al. (2007) utilisée dans le test de sensibilité/résistance de $C$. cassiicola aux fongicides a permis de classer les onze fongicides étudiés en cinq groupes selon leur pourcentage d'inhibition global de la croissance mycélienne de C. cassiicola (Tableau 3). Ainsi, le premier groupe de fongicides constitué de Pyraclostrobine + Fenpropimorphe, Iprodione, Carbendazime + Chlorothalonil et Carbendazime ont entrainé une forte inhibition de la croissance mycélienne de $C$. cassiicola dans l'ordre de 94 à $100 \%$. A faible dose de 10 ppm, C. cassiicola a montré une forte sensibilité à ces quatre fongicides. Corynespora cassiicola a donc été hautement sensible à ces 4 fongicides, qui se classent ainsi dans le premier niveau d'efficacité (très bon). Le deuxième groupe est composé de fongicides auxquels, $C$. cassiicola a été sensible. Il s'agit de Difénoconazole et Mancozèbe. Ces deux molécules ont inhibé la croissance mycélienne du champignon dans l'ordre respectif de 84,32 et 88,29\%. Ils se classent ainsi dans le groupe des fongicides ayant une bonne efficacité. Le troisième groupe est composé de Chlorothalonil et Triadiméfon auxquels $C$. cassiicola a montré une résistance modérée. Ces fongicides ont respectivement réduit la croissance 
mycélienne à des taux de 73,29 et 69,24\%, leur conférant de ce fait, une efficacité modérée. Le quatrième groupe, constitué uniquement d'Azoxyxtrobine a montré un faible niveau d'efficacité avec un pourcentage d'inhibition de la croissance mycélienne de l'ordre de 45,97\%. Quant au dernier groupe, il est constitué de Fosetyl - Aluminium et Métalaxyl + Oxyde de cuivre. Ils ont induit respectivement des pourcentages d'inhibition globaux de 30,34 et $12,82 \%$. Ainsi, C. cassiicola a été hautement résistant à ces deux molécules quelle que soit les doses utilisées et terminent au bas de l'échelle d'efficacité.

Figure 1 : Diamètre mycélien moyen de $C$. cassiicola en fonction des doses de chaque fongicide

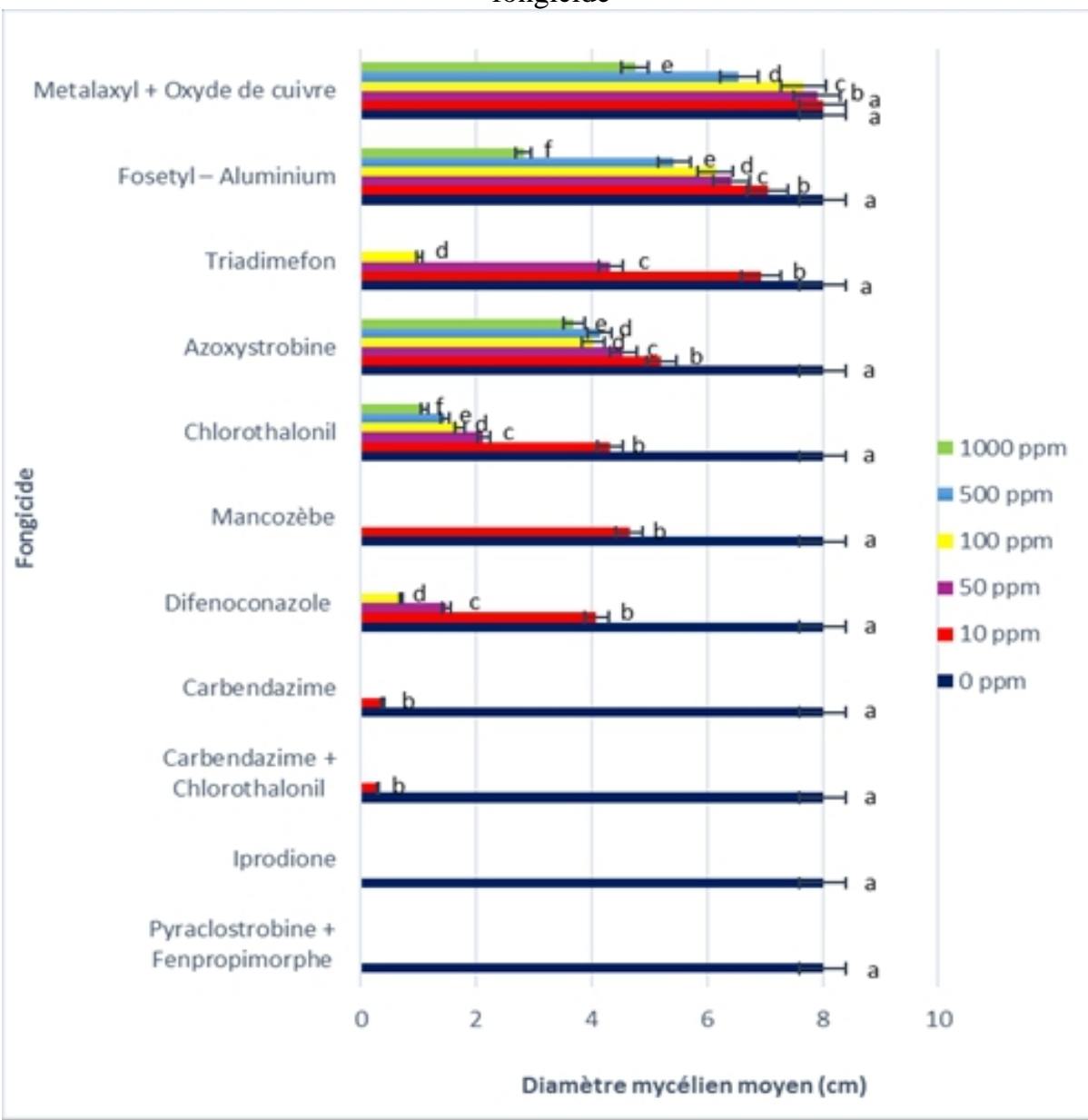

Au niveau de chaque fongicide, les barres portant la même lettre sont statistiquement identiques $(\alpha=5 \%)$ 
Tableau 3 : Pourcentage d'inhibition de la croissance mycélienne de $C$. cassiicola en fonction de la dose de chaque fongicide

\begin{tabular}{|c|c|c|c|c|c|c|}
\hline \multirow{2}{*}{ Fongicide } & \multicolumn{5}{|c|}{ Pourcentage d'inhibition (\%) à différentes doses $(\mathrm{ppm})$ des fongicides } & \multirow{2}{*}{$\begin{array}{l}\text { Pourcentage } \\
\text { d'inhibition } \\
\text { global (PIG) }\end{array}$} \\
\hline & 10 & 50 & 100 & 500 & 1000 & \\
\hline $\begin{array}{l}\text { Pyraclostrobine + } \\
\text { Fenpropimorphe }\end{array}$ & $100 \pm 0,00 \mathrm{a}$ & $100 \pm 0,00 \mathrm{a}$ & $100 \pm 0,00 \mathrm{a}$ & $100 \pm 0,00 \mathrm{a}$ & $100 \pm 0,00 \mathrm{a}$ & $100 \pm 0,00 \mathrm{a}$ \\
\hline Iprodione & $100 \pm 0,00 \mathrm{a}$ & $100 \pm 0,00 \mathrm{a}$ & $100 \pm 0,00 \mathrm{a}$ & $100 \pm 0,00 \mathrm{a}$ & $100 \pm 0,00 \mathrm{a}$ & $100 \pm 0,00 \mathrm{a}$ \\
\hline $\begin{array}{l}\text { Carbendazime + } \\
\text { Chlorothalonil }\end{array}$ & $94,50 \pm 0,05 b$ & $100 \pm 0,00 \mathrm{a}$ & $100 \pm 0,00 \mathrm{a}$ & $100 \pm 0,00 \mathrm{a}$ & $100 \pm 0,00 \mathrm{a}$ & $98,90 \pm 2,45 a$ \\
\hline Difenoconazole & $49,0 \pm 1,74 \mathrm{c}$ & $81,37 \pm 1,37 b$ & $91,25 \pm 1,02 b$ & $100 \pm 0,00 \mathrm{a}$ & $100 \pm 0,00 \mathrm{a}$ & $84,32 \pm 21,18 \mathrm{a}$ \\
\hline Mancozèbe & $41,47 \pm 1,35 d$ & $100 \pm 0,00 \mathrm{a}$ & $100 \pm 0,00 \mathrm{a}$ & $100 \pm 0,00 \mathrm{a}$ & $100 \pm 0,00 \mathrm{a}$ & $88,29 \pm 26,17 \mathrm{a}$ \\
\hline Chlorothalonil & $46,0 \pm 1,14^{\mathrm{e}}$ & $73,37 \pm 0,84 c$ & $78,62 \pm 1,09 d$ & $81,75 \pm 0,87 b$ & $86,25 \pm 0,09 b$ & $73,29 \pm 16,01 \mathrm{ab}$ \\
\hline Azoxystrobine & $34,87 \pm 0,92 f$ & $43,12 \pm 1,35 \mathrm{e}$ & $49,75 \pm 0,98$ & $48,25 \pm 1,20 \mathrm{c}$ & $53,87 \pm 0,09 d$ & $45,97 \pm 7,30 \mathrm{bc}$ \\
\hline Triadimefon & $13,0 \pm 1,46 \mathrm{~g}$ & $45,87 \pm 1,44 d$ & $87,37 \pm 1,09 \mathrm{c}$ & $100 \pm 0,00 \mathrm{a}$ & $100 \pm 0,00 \mathrm{a}$ & $69,24 \pm 38,50 \mathrm{ab}$ \\
\hline $\begin{array}{l}\text { Fosetyl - } \\
\text { Aluminium }\end{array}$ & $11,87 \pm 1,58 \mathrm{~h}$ & $19,75 \pm 0,98 f$ & $23,25 \pm 1,20 f$ & $32,12 \pm 1,02 \mathrm{~d}$ & $64,75 \pm 0,13 \mathrm{c}$ & $30,34 \pm 20,55 \mathrm{~cd}$ \\
\hline
\end{tabular}

Dans la même colonne, les valeurs portants les mêmes lettres sont statistiquement identiques (Newman et Keuls, $\alpha=0,05$ )

Un effet dose a été par ailleurs observé. Les résultats indiqués sur la figure 2 montrent que le pourcentage d'inhibition croît avec la concentration de fongicide. Ainsi, pour les doses de 10, 50, 100, 500 et $1000 \mathrm{ppm}$, les pourcentages d'inhibition ont été respectivement de 53,69, 75, 80 et $85 \%$. L'analyse statistique révèle en outre, une inhibition significative de la croissance mycélienne entre 10 et 100 ppm. Par contre, entre 500 et 1000 ppm, l'effet fongitoxique des fongicides n'est pas significatif.

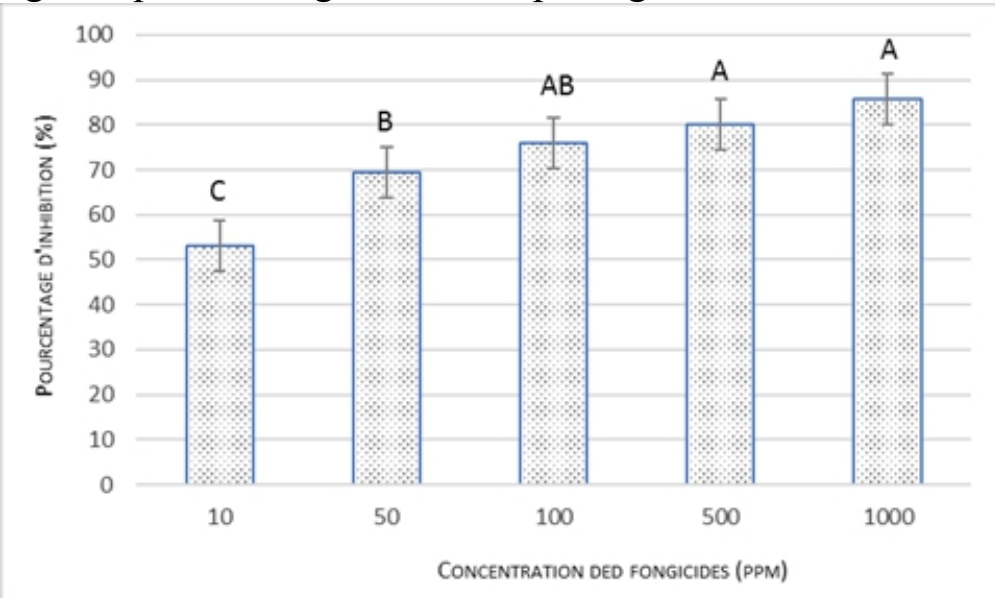

Figure 2 : Pourcentage d'inhibition de la croissance mycélienne de $C$. cassiicola en fonction des concentrations des fongicides

Les barres portant la même lettre sont statistiquement identiques $(\alpha=5 \%)$ 


\section{Discussion}

L'évaluation in vitro des fongicides a permis d'obtenir des résultats préliminaires et utiles sur l'efficacité des fongicides contre Corynespora cassiicola, agent fongique responsable de la maladie CLF. Dans cette étude, onze fongicides ont été testés à cinq différentes concentrations (10, 50, 100, 500 et 1000 ppm) contre C. cassiicola. Parmi ces fongicides, quatre à savoir le Pyraclostrobine + Fenpropimorphe, Iprodione, Carbendazime + Chlorothalonil et Carbendazime se sont avérés très efficaces. L'agent fongique a exprimé, même à faible dose (10 ppm), une hypersensibilité à ces quatre fongicides, surtout aux deux premiers avec une inhibition totale de leur développement. L'efficacité de Pyraclostrobine + Fenpropimorphe pourrait s'expliquer par la combinaison de deux matières actives. En effet, la Pyraclostrobine est une molécule systémique appartenant à la famille des strobilurines curatives à action endothérapique (Phytoma, 2003). Elle est caractérisée, de ce fait, par un champ d'activité très large, lui conférant un haut niveau d'efficacité. Son association avec le Fenpropimorphe, substance active de la famille des morphines, à mode d'action systémique serait à la base de l'inhibition totale du développement de $C$. cassiicola, donc de leur efficacité remarquable. Quant à l'Iprodione qui est un fongicide de contact de la famille des dicarboximides, son efficacité totale au laboratoire contre $C$. cassiicola serait due au fait qu'il inhibe la germination des spores et bloque le développement du mycélium (FRAC, 2018). Il agit, en effet, sur plusieurs sites comme les mitochondries, le réticulum endoplasmique et le noyau cellulaire pour les dénaturer. Le Carbendazime est une molécule couramment utilisée dans la lutte chimique contre les maladies cryptogamiques à cause de son effet fongitoxique sur les champignons. Fongicide systémique de la famille des benzimidazoles, il agit comme un inhibiteur du développement des tubes de germination et de la croissance de mycéliums (Acta, 2005) ; ce qui lui confère une très bonne efficacité contre $C$. cassiicola. Malgré son efficience, il est beaucoup utilisé en combinaison avec d'autre fongicide comme le Chlorothalonil, ce qui renforcerait davantage son efficacité. Des résultats similaires ont été rapportés par plusieurs auteurs tels que Vawdrey et al. (2008) ; Fernando et al. (2010) ; Manju (2011) et Avozani et al. (2014) à l'issu des tests d'efficacité fongicide au laboratoire. Il est, en effet ressorti de leur étude que certaines molécules telles que le Carbendazime, Chlorothalonil, Fenpropimorphe, Iprodione et Pyraclostrobine sont efficaces contre $C$. cassiicola. Le deuxième lot de fongicides ayant montré un bon niveau est constitué de Difénoconazole et Mancozèbe. Les autres fongicides, dans l'ordre d'efficacité moyenne à très faible, ont été Chlorothalonil, Triadimefon, Azoxyxtrobine, Fosetyl - Aluminium et Metalaxyl + Oxyde de cuivre.

Il convient de noter que tous les fongicides utilisés dans cette étude ayant deux matières actives associées, à l'exception de Metalaxyl + Oxyde de 
cuivre ont montré un bon niveau d'efficacité contre $C$. cassiicola. Cette efficience serait attribuable à leur spectre d'activité plus large du fait qu'ils sont constitués de la combinaison de deux substances actives. Des observations similaires ont été faites par Manju (2006) dans une étude sur les moyens de lutte chimique contre la défoliation causée par $C$. cassiicola sur les hévéas. Selon lui, la très bonne efficacité de Mancozèbe + Carbendazime serait due à sa double action (contact et systémique). En effet, les combinaisons de fongicides conduisent à une synergie car elles élargissent le spectre d'activité antifongique contre plusieurs maladies pouvant attaquer simultanément les cultures (Gisi, 1996). De plus, elles retardent la sélection de résistances individuelles dans les populations de pathogènes (Gisi, 1996). Cependant, la haute résistance de $C$. cassiicola au Métalaxyl + Oxyde de cuivre, malgré leur combinaison pourrait s'expliquer par le fait que le Métalaxyl est une molécule qui agit spécifiquement contre les champignons de la classe des Oomycètes (Acta, 2005). Il en est de même pour Fosetyl Aluminium, qui s'est aussi révélé moins efficace ; leur spécificité contre les Oomycètes serait à la base de leur inefficacité contre C . cassiicola (Acta, 2005) qui est un champignon de la classe des Ascomycètes. Les fongicides de contact utilisés dans ce test ont montré pour la plupart, un bon niveau d'efficacité contre $C$. cassiicola. Cette efficacité observée serait due à leur mode d'action multisite. En effet, ces substances chimiques ont la possibilité d'agir sur des sites variés, c'est-à-dire sur différents aspects de la physiologie d'un champignon ; ce qui empêche le pathogène de développer une résistance (Bacon 2002). Contrairement aux fongicides multisites, les fongicides unisites possèdent un seul site d'action ; ce qui pourrait favoriser à long terme, le développement de résistance comme l'a relevé Bacon (2002). Un effet dose a été également observé de 0 à 100 ppm. En effet, les fortes doses de fongicide ont entraîné des inhibitions significatives de la croissance mycélienne. Cependant, à une concentration seuil de 500 ppm, l'efficience des fongicides est stabilisée. Tous les fongicides, à dose élevée, ont certes entrainé des taux d'inhibitions significative de la croissance mycélienne de C. cassiicola comparativement au témoin, mais ils ne sont pas tous efficaces. En effet, l'efficacité d'un fongicide réside dans sa capacité à inhiber le développement du champignon à des doses relativement faibles ; et la dose efficace est celle à laquelle l'inhibition est totale (Sharma et Mohanan, 1991). Cela est tout à fait rationnel, dans la mesure où l'utilisation massive des fongicides entraînerait, non seulement, l'induction des souches de champignons résistantes mais aussi causerait irréversiblement une pollution environnementale avec ses multiples conséquences désastreuses. 


\section{Conclusion}

Dans la recherche de solution de lutte contre la maladie de chute des feuilles de l'hévéa (CLF) causée par Corynespora cassiicola, des travaux ont été menés au laboratoire. Les résultats des travaux ont révélé l'efficacité de quatre fongicides sur onze testés. Il s'agit dans l'ordre d'efficacité croissante de l'Iprodione (Piécho), Carbendazime + Chlorothalonil (Banko plus), Carbendazime (Carhino) et Pyraclostrobine + Fenpropimorphe (Comet plus). L'emploi de ces quatre formulations fongicides en plantation mature serait bien indiqué, pour venir à bout de cette pathologie foliaire.

\section{References:}

1. Acta. (2005). Index Phytosanitaire Acta, 41 : 205-344.

2. Avozani A., Reis E.M. \& Tonin R.B. (2014). Sensitivity loss by Corynespora cassiicola, isolated from soybean, to the fungicide carbendazim. SummaPhytopathologica, 40 (2): 273-276.

3. Awoderu V.A. (1969). A new leaf spot of para rubber Hevea brasiliensis in Nigeria. Plant Diseases reporter, 53 (5): 406-408.

4. Bacon R. (2002). Gestion de la résistance aux fongicides. Agriculture et Agroalimentaire Canada, 2002, 7 p.

5. Barthe P., Pujade-Renaud V., Breton F., Gargani D., Thai R., Roumestand C. \& De Lamotte F. (2007). Structure analysis of cassiicolin, a host-selective protein toxin from Corynespora cassiicola. Journal of Molecular Biology, 367: 89-101.

6. Fernando H.P.S., Jayasinghe C.K, Wijesundera R.L.C., Silva W.P.K. \& Nishanta E.A.D.N. (2010). Evaluation of screening methods against Corynespora leaf fall disease of rubber (Hevea brasiliensis). Journal of plant diseases and protection, 17 (1): 22-29

7. FRAC. (2018). FRAC Code List C. 2018: Fungicides sorted by mode of action (including FRAC Code numbering). Available at: http://www.phi-base.org/images/fracCodeList.pdf. Accessed on: May $31,2018$.

8. Georges M.K. \& Edathil T.T. (1980). Paper oresented at international rubber conference, 180, Kottayam, India.

9. Gisi U. (1996). Synergistic interaction of fungicides in mixtures. Phytopathology, 86: 1273-1279.

10. Jayasinghe C.K. (2000). Corynespora leaf fall: the most challenging rubber disease in Asian and African continents. Bulletin of the Rubber Research Institute of Sri Lanka, 42: 56-64.

11. Junqueira N.T.V., Gasparoto L., Morae V.H.F., Silva H.M. \& Lim T.M., (1985). New diseases caused by virus, fungus and also bacterium on rubber from Brazil and their impact on International Quarantine. In: Proceeding reginal Conference on plant quarantine support for 
agricultural development, Kuala Lumpur 10-12 Dec. 1985. ASEAN Plant quarantine center and training institute. pp. 253-260.

12. Kajornchaikul P. (1987). Corynespora disease of hevea in Thailand, pp. 1-5. In Proceedings of IRRDB Symposium on pathology of hevea brasiliensis. Chiangmai.

13. Kumar S.A., Reddy E.P.N., Reddy H.K. \& Devi C.M. (2007). Evaluation of fungicidal resistance among Colletotrichum gloesporioides isolates causing mango anthracnose in Agri export zone of Andhra Pradesh, India. Plant Pathology bulletin, 16:157-160.

14. Liyanage A.D.E.S, Jayasinghe C.K, Liyanage N.I.S \& Jayaratne R. (1986). Corynespora leaf spot disease of rubber (Hevea brasilensis): a new record. Journal of the Rubber Research Institute of Sri Lanka, 65: 47-50.

15. Manju M.J., Idicula S.P., Jacob C.K., Vinod K.K., PREM E.E., Suryakumar M. \& Kothandaraman R. (2001). Incidence and severity of Corynespora Leaf Fall (CLF) disease of rubber in coastal Karnataka and North Malabar region of Kerala. Indian Journal of Natural Rubber Research, 14: 137-141.

16. Manju M.J., Idicula S.P., Jacob C.K., Vinod K.K., PREM E.E. \& Suryakumar M. (2006). Chimical control of Corynespora leaf fall disease. In : Corynespora leaf disease of Hevea brasiliensis Strategies for management. (Editor Kuruvilla Jacob C.) pp 102 - 108. (Rubber Research Institute of India).

17. Newsam A. (1961). Rubber Research Institute of Malaysia, Pathology Division Report, 63-70.

18. Phytoma. (2003). Qu'est-ce que la pyraclostrobine ? Une strobilurine à part. Récupéré le 01 juin 2018 de http://phytoma-ldv.com/article23120.

19. Pongthep K. (1987). Corynespora disease of Hevea in Thailand. IRRDB's symposium on pathology of Hevea in Chieng Mai, Thailand (Eds. JHKKo, S Chow, KH Han) pp.16-22. (Rubber Research Institute of Thailand: Bangkok).

20. Ramakrishnan T.S. \& Pillay P.N.R. (1961). Leaf spot of rubber caused by Corynespora cassiicola (Berk \& Curt) Wei. Rubber board bulletin, 5: $52-53$.

21. Sharma J.K. \& Mohanan C. (1991). In vitro evaluation of fungicide against Cylindrocladium spp. causing diseases of Eucalyptus in Kerela, India. European Journal of Forest Pathology, 21: 17-26.

22. Teoh C.H. (1983). Corynespora leaf fall of Hevea in West Java. Malaysian Plant Protection Society Newsletter 7, 12-13.

23. Vawdrey L.L., Grice K.R.E. \& Westerhuis D. (2008). Field and laboratory evaluations of fungicides for the control of brown spot 
(Corynespora cassiicola) and black spot (Asperisporium caricae) of papaya in far north Queensland, Autralia. Australasian plant pathology, 37: 552-558

24. Vincent J.M. (1927). Distortion of fungal hyphae in the presence of certain inhibitors. Nature, $850 \mathrm{pp}$.

25. Wahounou P.J., Canh T.V., Keli J.Z. \& Eschbach J. M. (1996.) Development of Corynespora cassiicola and Colletotrichum gloeosporioides leaf fall disease in rubber plantation in Africa. Proceeding Workshop on Corynespora Leaf Fall Disease of Hevea Rubber, pp 99-107. (Indonesian Rubber Research Institute). 